\title{
Invasive Meningococcal Disease Unraveling a Novel Mutation in the C5 Gene in a Portuguese Family
}

\author{
Filipa Marujo, MD, * Luís Carlos Costa, MD, * Regina Duarte, MD, † Maria João Brito, MD, $\neq$ \\ Ana Cordeiro, $M D,{ }^{*}$ Conceição Neves, $M D, *$ and João Farela Neves, $M D^{*}+\S$
}

\begin{abstract}
Although bacterial meningitis is a rare presentation of a congenital immunodeficiency, invasive meningococcal disease is classically associated with complement deficiencies. We report a patient from a consanguineous kindred presenting with an invasive meningococcal disease caused by serogroup B meningococcus that revealed an underlying C5 deficiency caused by a novel mutation in the $\mathrm{C} 5$ gene.
\end{abstract}

Key Words: invasive meningococcal disease, Neisseria meningitides, complement component deficiencies, C5 deficiency, p.Tyr352Cys

(Pediatr Infect Dis J 2019;38:416-418)

nvasive meningococcal disease (IMD) is caused by Neisseria meningitidis, a common commensal member of the human upper respiratory tract and a major cause of bacterial meningitis and septicemia. ${ }^{1}$ Twelve serogroups of this Gram-negative have been identified, of which six (A, B, C, W-135, Y and X) are responsible for the majority of IMD cases worldwide. ${ }^{1}$ Serogroup B (MenB) and C (MenC) meningococci are responsible for the majority of the burden of the disease in Europe, and the implementation of appropriate meningococcal immunization programs have led to decreasing incidence. ${ }^{2}$ Accordingly, the incidence of IMD in Portugal has been declining over the past decade, in part because of the high coverage rate achieved after the implementation of the MenC vaccine in the national immunization program in 2006. ${ }^{2}$ Nevertheless, and even before the generalization of vaccination against MenC, MenB has been the most prevalent cause of IMD in Portugal, demonstrating the importance of high coverage rates against MenB. ${ }^{2}$

Primary immunodeficiencies (PIDs) are a heterogenous group of monogenic disorders that affect the development, function, or both, of the immune system. ${ }^{3}$ PIDs can present as recurrent, unusual or persistent infection as frequent, recurrent or persistent infection, but also as precocious or severe autoimmunity and inflamation. ${ }^{3}$

Most infections with $N$. meningitidis are seen in patients without known predisposing factors. Presence of personal history of recurrent and/or invasive infections, family history of meningitis or severe infections or unexplained family childhood deaths

Accepted for publication June 14, 2018

From the *Primary Immunodeficiencies Unit, Hospital Dona Estefânia- CHLC, EPE, †Plastic Surgery Unit, Hospital Dona Estefânia- CHLC, EPE, †̣Infectious Diseases Unit, Hospital Dona Estefânia- CHLC, EPE, and §CEDOC, Chronic Diseases Research Center, NOVA Medical School, Lisboa, Portugal. The authors have no funding or conflicts of interest to disclose.

F.M. (medical doctor of the patient) wrote the paper; L.C.C, R.D., M.J.B., A.C., C.N., (medical doctor of the patient) reviewed the article; and J.F.N. (medical doctor of the patient) designed the research study and reviewed the article.

Address for correspondence: Filipa Marujo, MD, Primary Immunodeficiencies Unit, Hospital Dona Estefania, Pediatric University Unit, Rua Jacinta Marto, 1169-045 Lisbon, Portugal. E-mail: filipa.marujo10@gmail.com or filipa. marujo@chlc.min-saude.pt.

Supplemental digital content is available for this article. Direct URL citations appear in the printed text and are provided in the HTML and PDF versions of this article on the journal's website (www.pidj.com).

Copyright (C) 2018 Wolters Kluwer Health, Inc. All rights reserved.

ISSN: 0891-3668/19/3804-0416

DOI: $10.1097 /$ INF.0000000000002149 are suggestive of PID as an underlying cause. History of asplenia, treatment with eculizumab are other causes for $N$. meningitidis infections. ${ }^{4,5}$ The timely diagnosis of these conditions is crucial for the long-term prognosis of these patients. ${ }^{4}$

Screening for a complement deficiency is classically performed on patients suffering from recurrent IMD and on those with an uncommon $N$. meningitidis serogroup. ${ }^{4-6}$

We report a patient with IMD who was screened for complement deficiency because of parental consanguinity, leading to the diagnosis of the first Portuguese kindred with C5 deficiency caused by a novel $C 5$ gene mutation.

\section{CASE REPORT}

A 6-year-old girl was admitted to the emergency room because of persistent vomiting. She was discharged but returned 10 hours later with generalized suffusions (Fig., Supplemental Digital Content 1, http://links.lww.com/INF/D227). Her personal medical history was unremarkable. Her parents were first-degree cousins, just like her grandparents, and there is a history of multiple deaths after an infection at an early age (Fig. 1).

At physical examination, the patient was oriented but hypotensive (mean arterial pressure, $30 \mathrm{mmHg}$ ), with severe generalized purpura fulminans (in the trunk, legs and arms). She had no neck stiffness, and no papilledema was observed. Laboratory investigations are shown in Fig., Supplemental Digital Content 2, http:// links.lww.com/INF/D228.

The patient promptly received ceftriaxone $(100 \mathrm{mg} / \mathrm{kg} / \mathrm{d})$ and prophylaxis with rifampicin was given to the intimate contacts. The patient required fluid resuscitation, then was transferred to pediatric intensive care unit (PICU). In PICU, she required aminergic support with dopamine. With the persistence of septic shock, she was intubated, ventilated and received epinephrine.

$N$. meningitidis grew in the blood culture (later the N. meningitidis was characterized by polymerase chain reaction as being serogroup B). Lumbar puncture was delayed until the third day of hospitalization because of coagulopathy. Cerebrospinal fluid was clear, and the cell count, proteins and glucose were within normal range. Polymerase chain reaction for $N$. meningitidis was negative in the cerebrospinal fluid.

She was withdrawn from mechanical ventilation after 2 days, the aminergic support was stopped after 5 days and she was transferred from the PICU to the Infectious Diseases Unit after 6 days. The patient recovered completely with minor cutaneous sequelae for which she received treatment by plastic surgery.

The presence of parental consanguinity prompted investigation for an underlying complement deficiency. She had absent CH100 activity and very low C5 (Fig. 1). A 10-year-old sister also had an undetectable $\mathrm{CH} 100$ with reduced levels of C5. Sanger sequencing of the $\mathrm{C} 5$ gene revealed the presence of a novel mutation (p.Tyr352Cys) in homozygosity in both the patient and her sister. Bioinformatics analysis predicted that it affected a highly conserved residue and that it was pathogenic (SIFT Sorting Intolerant From Tolerant: 0.98, deleterious; polyphen: 0.93 , probably damaging; mutation taster: disease causing). The parents were heterozygous for the same mutation. 


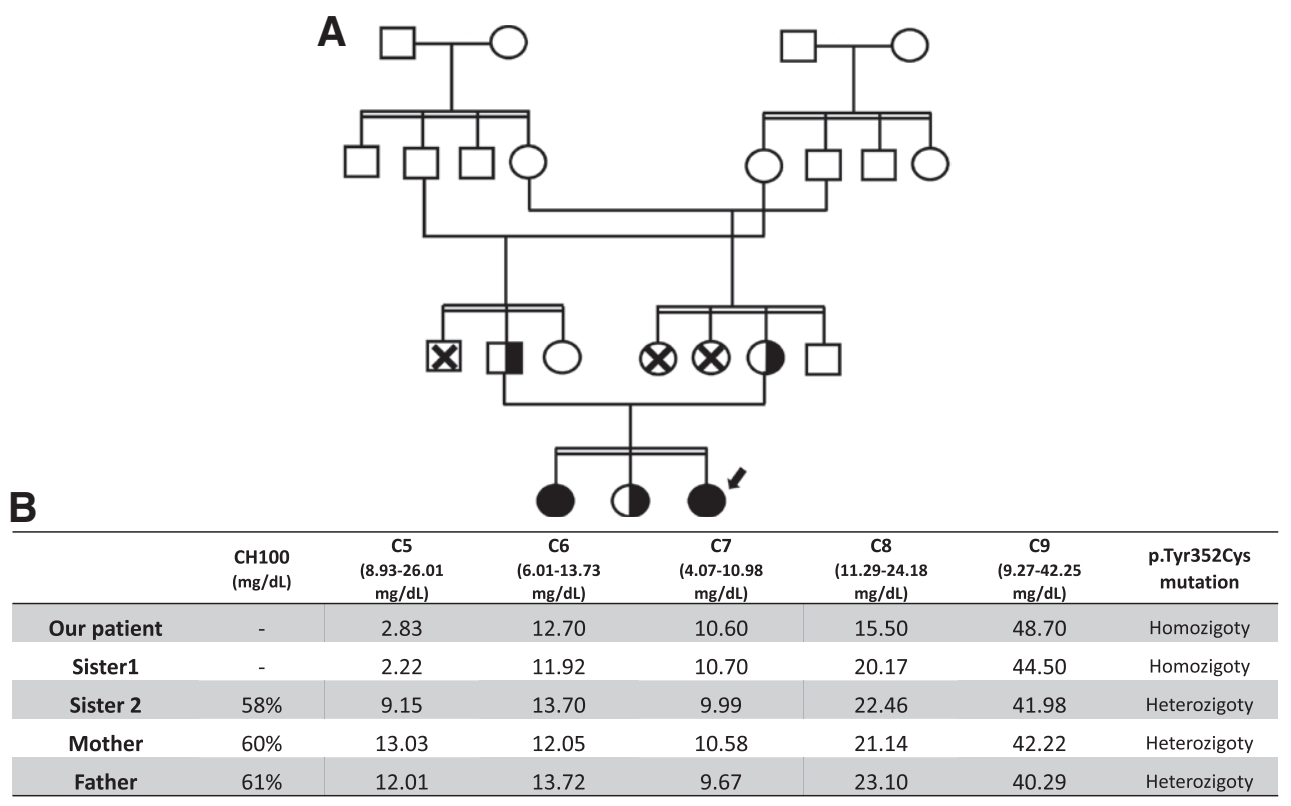

FIGURE 1. A: Family tree showing family consanguinity and the homozygous and heterozygous patients. " $X$ ": dead family members. B: Concentration of complement proteins and mutation status in family members.

The patient and the asymptomatic sister were immunized with the meningococcal quadrivalent conjugate (ACWY135) vaccine and the 4CMenB vaccine (Bexsero, https://gsksource.com/ pharma/content/dam/GlaxoSmithKline/US/en/Prescribing_Information/Bexsero/pdf/BEXSERO.PDF), as well as with conjugate and polysaccharide pneumococcal vaccines.

\section{DISCUSSION}

The complement system is an ancient proinflammatory and microbial destruction system that may be considered part of both the innate and adaptive systems..$^{6-8}$ It consists of the classical, alternative and lectin-binding pathways. One of the goals of the activation of the different pathways of complement is the deposit of $\mathrm{C} 3 \mathrm{~b}$ on the target recognized as foreign and later activation of the membrane attack complex (MAC).,7,8 The MAC is a multimolecular unit composed of the terminal components of complement (C5-C9) that increases the immune response, resulting in opsonization of the target particle. ${ }^{7,8}$ The MAC forms a pore that allows the rapid influx of ions into the bacterial cell and, thanks to the influx of water and swelling, rupture of the cell occurs by osmotic lysis. ${ }^{7,8}$

The MAC cannot be formed if any of components are absent. The role of the complement system in innate immune defenses against IMD is well established, and individuals deficient in components of the terminal complement pathways are highly predisposed to invasive, often recurrent, meningococcal infections. ${ }^{8}$ $N$. meningitidis has been the microorganism responsible for $75 \%-85 \%$ of the infections identified in these patients. ${ }^{4,7}$ The infection by meningococcus has been associated with deficiency of any of the plasmatic proteins of complement, but the evidence has shown it more commonly involves deficiency of the terminal components of the complement pathway ( $40 \%$ presented at least one episode). ${ }^{5,9}$ In fact, the association between C5 deficiency and Neisseria infection may be explained by a crucial role of $\mathrm{C} 5$ in bacterial killing of $N$. meningitidis recently described in a whole blood in vitro model. ${ }^{9}$

Despite the high risk for meningococcal disease, there is a 5to 10 -fold decrease in the probability of death because of this disease in patients with complement deficiency, when compared with the general population. ${ }^{7}$ It looks like the same condition that predisposes to infection could protect against the lethal consequences of the disease, because the inability to generate a functionally active MAC leads to reduced microbial damage and less severe endotoxin shock. ${ }^{7,9}$ This hypothesis is supported by Lappegård et $\mathrm{al}^{10}$, who have shown that C5 is essential for the Gram-negative bacteria killing and for the huge inflammatory response induced by the bacteria. Ward also suggested that activated products of $\mathrm{C} 5$ and its binding to C5a-receptor would be responsible for loss of immune innate functions and development of septic shock. ${ }^{11}$ This fact suggests that an exacerbated response from the host is the main factor associated with the clinical manifestations and the outcome of the disease and also that the lack of C5 may protect from the most serious consequences of meningococcal sepsis. ${ }^{1,12}$

It is well known that IMD can be the first and only manifestation of complement deficiency, and patients are usually asymptomatic until the first IMD takes place (many patients have the first episode in adulthood). Although it is difficult to decide when to investigate the presence of a predisposing factor, screening is classically warranted in the case of family history of IMD, recurrent IMD infections or when IMD is caused by an uncommon serogroup., ${ }^{4,7}$ We propose that patients presenting IMD and with parental consanguinity should also be screened for complement deficiency.

In patients with IMD and known risk factors, the assessment of the complement function (total complement activity or CH50 or CH100) should be quantified. ${ }^{6}$ This is a screening assay for the activation of the classical complement pathway based on a hemolytic assay in which an immune complex is formed by adding antibodies that react with a surface antigen on sheep red blood cells. ${ }^{6}$ When sheep red blood cells are incubated with test serum, the classical pathway of complement is activated resulting in hemolysis. ${ }^{6}$ When the complement function is altered, further evaluation of the components of the terminal pathway of complement (C5-C9) must be considered. ${ }^{6,7}$

The proper diagnosis of complement defects in a patient with IMD is crucial not only for prevention of recurrent infection in the propositus but also for the screening of asymptomatic family members and for genetic counselling. ${ }^{13}$ Patients with MAC deficiency should be vaccinated against encapsulated bacteria, such as 
N. meningitidis, Streptococcus pneumonia and Haemophilus influenza type $b .^{5,12}$

In summary, we report the first 2 Portuguese patients with C5 deficiency caused by a novel mutation in the C5 gene that was diagnosed after a IMD caused by MenB in a consanguineous family.

\section{REFERENCES}

1. Grimnes G, Beckman H, Lappegård KT, et al. Recurrent meningococcal sepsis in a presumptive immunocompetent host shown to be complement C5 deficient-a case report. APMIS. 2011;119:479-484.

2. Simões MJ, Bettencourt C, De Paola R, et al. Predicted strain coverage of a meningococcal multicomponent vaccine (4CMenB) in Portugal. PLoS One. 2017; 12:e0176177.

3. Notarangelo LD. Primary immunodeficiencies. J Allergy Clin Immunol. 2010;125(2 suppl 2):S182-S194.

4. Overtuf GD. Indications for the immunological evaluation of patients with meningitis. Clin Infect Dis 2003; 36:189-194.

5. Colobran R, Franco-Jarava C, Martín-Nalda A, et al. Novel mutations causing c5 deficiency in three north-african families. J Clin Immunol. 2016;36:388-396
6. Frazer-Abel A, Sepiashvili L, Mbughuni MM, et al. Overview of laboratory testing and clinical presentations of complement deficiencies and dysregulation. Adv Clin Chem. 2016;77:1-75.

7. Figueroa JE, Densen P. Infectious diseases associated with complement deficiencies. Clin Microbiol Rev. 1991;4:359-395.

8. Lewis LA, Ram S. Meningococcal disease and the complement system. Virulence. 2014;5:98-126.

9. Schejbel L, Fadnes D, Permin H, et al. Primary complement C5 deficiencies - molecular characterization and clinical review of two families. Immunobiology. 2013;218:1304-1310.

10. Lappegård KT, Christiansen D, Pharo A, et al. Human genetic deficiencies reveal the roles of complement in the inflammatory network: lessons from nature. Proc Natl Acad Sci U S A. 2009;106:15861-15866.

11. Ward PA. Role of the complement in experimental sepsis. J Leukoc Biol 2008; 83:467-470.

12. Arnaout R, Al Shorbaghi S, Al Dhekri H, et al. C5 complement deficiency in a Saudi family, molecular characterization of mutation and literature review. J Clin Immunol. 2013;33:871-875.

13. Rosain J, Hong E, Fieschi C, et al. Strains responsible for invasive meningococcal disease in patients with terminal complement pathway deficiencies. J Infect Dis. 2017;215:1331-1338.

\section{Current Abstracts}

\section{Edited by Robert J. Leggiadro, MD}

\section{Multistate Infestation With the Exotic Disease-vector \\ Tick Haemaphysalis longicornis-United States, August 2017 to September 2018}

Beard CB, Occi J, Bonilla DL, et al. MMWR Morbid Mortal Wkly Rep. 2018; 67:1310-1313

Haemaphysalis longicornis is a tick indigenous to eastern Asia and an important vector of human and animal disease agents, resulting in such outcomes as human hemorrhagic fever and reduction of production in dairy cattle by $25 \%$. H. longicornis was discovered on a sheep in New Jersey in August 2017. This was the first detection in the United States outside of quarantine. In the spring of 2018, the tick was again detected in the index site, and later, in other counties in New Jersey, in 7 other states in the eastern United States and in Arkansas. The hosts included 6 species of domestic animals, 6 species of wildlife and humans.

H. longicornis is native to eastern China, Japan, the Russian Far East and Korea. It is an introduced, and now established, exotic species in Australia, New Zealand and several island nations in the western Pacific Region. Where this tick exists, it is an important vector of human and animal disease agents. In China and Japan, it transmits the severe fever with thrombocytopenia syndrome virus, which causes a human hemorrhagic fever, and Rickettsia japonica, which causes Japanese spotted fever. Studies in Asia identified ticks infected with various species of Anaplasma, Babesia, Borrelia, Ehrlichia, and Rickettsia, and all of these pathogen groups circulate zoonotically in the United States. In addition, parthenogenetic reproduction, a biologic characteristic of this species, allows a single introduced female tick to generate progeny without mating, thus resulting in massive host infestations. In some regions of New Zealand and Australia, this tick can reduce production in dairy cattle by $25 \%$.

To address this potential infectious disease threat, the U.S. Department of Agriculture Animal and Plant Inspection Service coordinated cooperative enhanced surveillance efforts through telephone conference calls with local, state and federal agricultural and public health agencies. Through these efforts, enhanced vector and animal surveillance was implemented to detect additional tick infestations in the United States. Suspect archival specimens that were available among previously collected ticks were also examined.

From August 2017 to September 2018, vector and animal surveillance efforts resulted in 53 reports of H. longicornis in the United States, including $38(72 \%)$ from animal species [23 (61\%) from domestic animals, $13(34 \%)$ from wildlife, and $2(5 \%)$ from humans], and $15(28 \%)$ from environmental sampling of grass or other vegetation using cloth drags or flags or carbon dioxide-baited tick traps. With the exception of one report from Arkansas, the remaining reports of positively identified ticks are from 8 eastern states: New Jersey $(16,30 \%)$, Virginia $(15,28 \%)$, West Virginia $(11,21 \%)$, New York (3, 6\%), North Carolina (3, 6\%), Pennsylvania (2, $4 \%)$, Connecticut (1,2\%) and Maryland (1, 2\%).

Surveillance efforts did not include testing the ticks or hosts for pathogens. No cases of illness in humans or other species were reported. Concurrent reexamination of archival historical samples showed that invasion occurred years earlier. Ticks collected from a deer in West Virginia in 2010 and a dog in New Jersey in 2013 were retrospectively identified as H. longicornis.

Comment: The biology and ecology of H. longicornis as an exotic species in the United States should be characterized in terms of its vector competence (ability to transmit a pathogen) and vectorial capacity (feeding habits, host preference, climatic sensitivity, population density and other factors that can affect the risk for pathogen transmission to humans) for tickborne pathogens known to be present in the United States. Where $H$. longicornis is detected, there should be testing for a range of indigenous and exotic viral, bacterial and protozoan tickborne pathogens potentially transmitted by $H$. longicornis. Given the similarity between severe fever with thrombocytopenia syndrome virus and Heartland virus, a tickborne phlebovirus, further evaluation of the potential role of $H$. longicornis in transmission of this disease agent among animal reservoirs and possibly to humans is warranted. 